\title{
Muonium avoided level crossing measurement of electron spin relaxation rate in a series of substituted anthradithiophene based molecules
}

\author{
S. Han ${ }^{\text {a,b }}$, K. Wang ${ }^{\text {a,b }}$, M. Willis ${ }^{\text {a }}$, L. Nuccio ${ }^{c}$, F.L. Pratt ${ }^{d}$, J.S. Lord $^{\text {d }}$, K.J. Thorley ${ }^{\mathrm{e}}$, \\ J. Anthony ${ }^{\mathrm{e}}$, A.J. Drew ${ }^{\mathrm{a}, \mathrm{b}, \mathrm{d}}$, S. Zhang ${ }^{\mathrm{a}, \mathrm{b}}$, L. Schulz ${ }^{\mathrm{a}, *}$ \\ a Sichuan University, School of Physical Science and Technology, Chengdu, Sichuan 610064, China \\ ${ }^{\mathrm{b}} \mathrm{Queen}$ Mary University of London, Mile End Road, London E1 4NS, UK \\ ${ }^{\mathrm{c}}$ Department of Physics and Fribourg Centre for Nanomaterials-Frimat, University of Fribourg, Chemin du Musée 3, CH-1700 Fribourg, Switzerland \\ d ISIS Muon Facility, Rutherford Appleton Laboratory, STFC, Harwell Science Campus, Chilton, Didcot OX11, UK \\ e Department of Chemistry, University of Kentucky, Lexington, Kentucky 40506-0055, USA
}

Muon spin spectroscopy and in particular the avoided level crossing technique is introduced, with the aim of showing it as a very sensitive local probe for electron spin relaxation in organic semiconductors. Avoided level crossing data on tert-butyl-ethynyl anthradithiophene, tri-methyl-silyl-ethynyl anthradithiophene and tri-ethygermyl-ethynyl anthradithiophene at different temperatures are presented. This series of molecules have an identical anthradithiophene backbone, but we have performed a targeted substitution on the central atom of the two side groups, of C, Si and Ge. We extracted the electron spin relaxation for the three molecules of this series and discuss them in the context of previously published results.

\section{Introduction}

Organic semiconductors are extremely promising materials for spintronics thanks to their long spin coherence times. However, spin phenomena in these materials are not yet fully understood, partly due to the lack of suitable characterisation techniques, since those extensively used to characterise inorganic semiconductors are not always applicable to organic materials [1-3]. Muon spin relaxation ( $\mu \mathrm{SR})$ has been proven to be an applicable probe in this field, as it is sensitive to spin dynamics in organic semiconductors on a molecular length scale [4-7]. In organic semiconductors the implanted muons form a hydrogen-like system, called muonium, by capturing an electron. In muonium the muon and electron spins are coupled through hyperfine interaction. As a consequence relaxation on the electron spin can induce changes in the muon spin, which can be detected. By performing targeted chemical

\footnotetext{
Corresponding author. Tel. +86 18180519891.

E-mail addresses: sijie.zhang@scu.edu.cn (S. Zhang), lschulz@scu.edu.cn (L. Schulz)
}

substitutions, it is then possible to identify the underlying mechanism of electron spin relaxation in the series of molecules, which has been a matter of debate for some time [5].

\section{Muon spin relaxation and avoided level crossing}

In $\mu$ SR experiments, $100 \%$ spin polarised muons are implanted in the sample, and as part of its thermalisation process in the material, it takes part in a series of electron capture and loss processes, thermalising as an interstitial positive muon or as a hydrogen-like species called muonium ( $\mathrm{Mu}$ ) [8-10]. This latter species can then chemically bind to an unsaturated organic molecule, in a similar way to hydrogenation, and is an extremely sensitive probe of spin dynamics intrinsic to the molecule [5-10]. If an external magnetic field is applied in the direction of the initial muon spin, as it is in so called longitudinal field experiments, the Hamiltonian for muonium can be written as

$H=-\gamma_{\mu} \mathrm{h} \boldsymbol{I} \times \boldsymbol{B}+\gamma_{\mathrm{e}} \mathrm{h} \boldsymbol{S} \times \boldsymbol{B}+\mathrm{h} \boldsymbol{S} \times \boldsymbol{A} \times \boldsymbol{I}$

where $\gamma_{\mu}$ and $\gamma_{\mathrm{e}}$ are the muon and electron gyromagnetic ratios, $\boldsymbol{B}$ is the applied longitudinal field, $\boldsymbol{I}$ and $\boldsymbol{S}$ are the muons and 


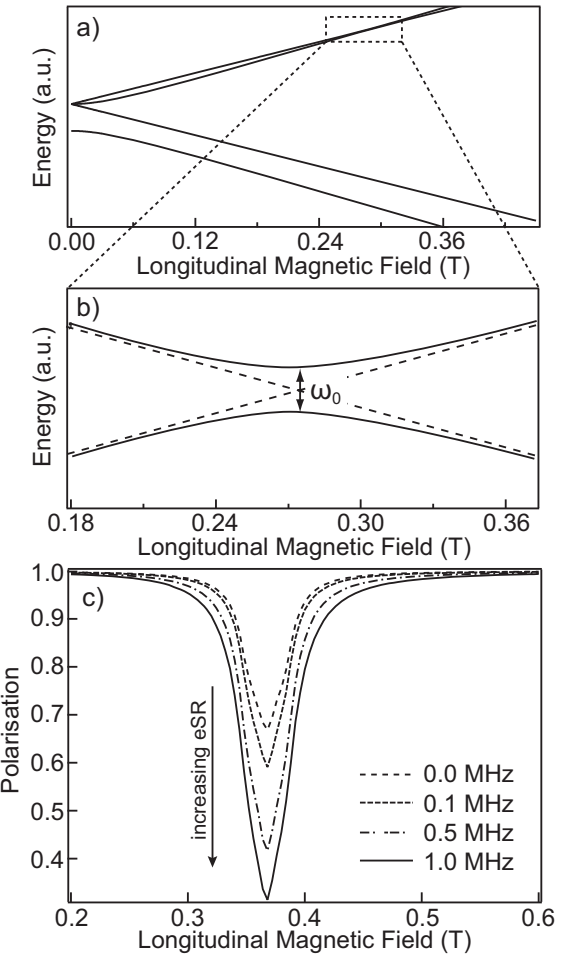

Fig. 1. (a) The muonium Breit-Rabi diagram with a hyperfine coupling constant of $A=80 \mathrm{MHz}$. (b) Zoom-in region of the energy levels near the ALC. (c) Simulated muon polarization for several different values of electron spin relaxation rate.

electrons spins and $\boldsymbol{A}$ is the hyperfine tensor [10]. The energy levels as a function of the applied magnetic field that originates from this Hamiltonian for an isotropic hyperfine interaction are shown in the Breit-Rabi diagram in Fig. 1(a).

At high enough fields the electron and muon spins are decoupled and the state of the system can be described by products of pure Zeeman states for the electron and muon. At a high field which depends on the value of the hyperfine coupling constant, two of the levels should cross, as can be observed in Fig. 1(b) (dashed lines). For a Hamiltonian $H=H_{0}+H^{\mathrm{I}}$ where $H^{\mathrm{I}}$ can be treated as a perturbation (for example, from interactions with nuclei or cross-relaxation effects), the correction to the energies up to second order is given by

$E_{n}-E_{n}^{0}=\left\langle\Psi_{n}\left|H^{I}\right| \Psi_{n}\right\rangle+\sum_{m} \frac{\left|\left\langle\Psi_{n}\left|H^{I}\right| \Psi_{n}\right\rangle\right|^{2}}{E_{n}^{0}-E_{m}^{0}}$

where $E_{n}^{0}$ and $\Psi_{n}$ are the n-th zero order energy and eigenstate and the denominator becomes tiny when the two unperturbed levels approach $[9,10]$. The effect of this correction to the energy levels is to avoid the crossing (see Fig. 1(b)). Close to this avoided level crossing (ALC), the states are no longer pure Zeeman states, but they are instead mixtures of states corresponding to different values of the muon magnetic quantum number. As a consequence a loss of polarisation of the muon can be observed [8,9]. ALCs can be classified by the change in total quantum number $M$. If the hyperfine interaction is isotropic, the most commonly observed ALC is the one with $\Delta M=0$, that corresponds to a muon-nuclear spin flip-flop. However in solids the most intense crossing is $\Delta M=1$, corresponding to a muon spin flip and is observed only when anisotropy in the interaction is present. $\Delta M=2$ transitions exist, but they are usually very weak and narrow, and are generally not observed experimentally [10]. A simulation of the muon's polarisation through this avoided crossing, from here on in termed an ALC curve, is shown in Fig. 1(c). The shape and position of this ALC curve depends on the hyperfine coupling constants (in solids, both isotropic and anisotropic). However, the presence of an electron spin relaxation rate (eSR) also has an effect on the ALC curve, as can be seen in Fig. 1(c). For low values of the eSR $(\leq 5 \mathrm{MHz}$ ), the ALC position with respect to the $x$ axis (magnetic field) and the general ALC shape do not change as a function of the eSR but the ALC amplitude (loss of polarisation) enormously increases. We have modelled the effect of eSR through a density matrix formalism, defined as

$\rho(t)=\frac{1}{4}\left[1+\boldsymbol{p}(t) \times \boldsymbol{\sigma}+\boldsymbol{p}_{\mathrm{e}}(t) \times \boldsymbol{\tau}+\sum_{\mathrm{j}, \mathrm{k}} P^{\mathrm{j} \mathrm{k}}(t) \sigma^{\mathrm{j}} \tau^{\mathrm{k}}\right]$

where $\boldsymbol{p}(t), \boldsymbol{p}_{\mathrm{e}}(t)$ and $P^{\mathrm{jk}}(t)$ are the muon, electron and mixed polarisations, respectively. The time evolution of $\rho(t)$ is described by the equation

$i \mathrm{~h} \frac{\mathrm{d} \rho}{\mathrm{d} t}=[H, \rho]$

from which the time dependent polarisations can be obtained [11]. The general solution can only be determined numerically. If an electron spin relaxation mechanism is present in the system its effect can be taken into account phenomenologically by introducing extra terms $-\lambda p_{\mathrm{e}}$ and $-\lambda P^{\mathrm{jk}}$ in the differential equations for the polarisations obtained from Eq. (4) [12]:

$\frac{\mathrm{d} \boldsymbol{p}_{e}}{\mathrm{~d} t}=\ldots-\lambda p_{e}, \frac{\mathrm{d} P^{\mathrm{jk}}}{\mathrm{d} t}=\ldots-\lambda P^{\mathrm{jk}}$

\section{Experimental method}

Tert-butyl-ethynyl anthradithiophene (TBu-ADT), tri-methylsilyl-ethynyl anthradithiophene (TMS-ADT) and tri-ethygermylethynyl anthradithiophene (TGe-ADT) were synthesized and purified according to a published procedure $[13,14]$ and were purified by repeated recrystallisation from dichloromethane/ ethanol. The only change in the chemical structure is the substitution of the central atom in the side-group with C, Si and

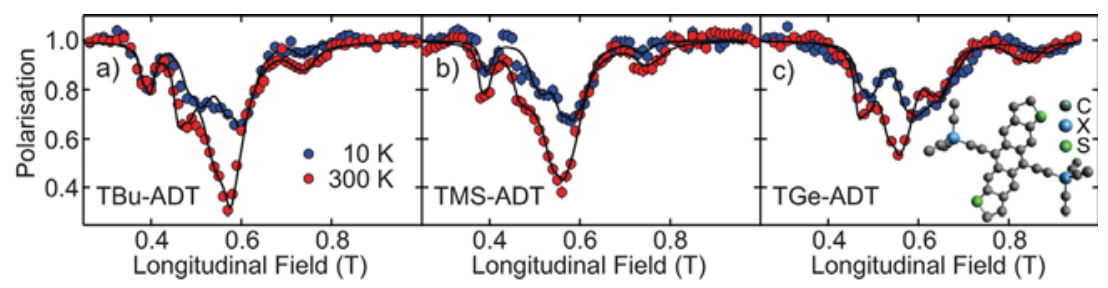

Fig. 2. The ALC resonances for (a) TBu-ADT, (b) TMS-ADT and (c) TGe-ADT for $10 \mathrm{~K}$ and $300 \mathrm{~K}$, with the associated modelling to extract the eSR and hyperfine constants. The molecular structure is shown in the inset of (c). Hydrogen is omitted for clarity. Only the central atom in the side-group modified (blue). (For interpretation of the references to colour in this figure legend, the reader is referred to the web version of this article.) 
Ge. They each share a common back-bone, which is a number of fused rings mainly comprising carbon and hydrogen, with the exception being the end rings, which contain $S$. Their structures are shown in the inset of Fig. 2(c). Analogous to the previously published work performed on the substituted triethylsilylethynyl (TES) and metal hydroxyquinolate (Xq3) series [5], performing targeted chemical substitutions can in principle identify the underlying electron spin relaxation mechanism.

Muon experiments were performed at the HIFI spectrometer at ISIS, Rutherford Appleton Laboratory. About $200 \mathrm{mg}$ of the three materials were placed in an envelope $(17 \mathrm{~mm} \times 17 \mathrm{~mm})$ made of $25 \mu \mathrm{m}$ thick silver foil (99.99\% pure). Another layer of silver foil was placed in front of the sample as a degrader, to assure that the muons stop in the sample. The software Wimda [15] was used to extract the time-integrated data and plot the ALCs as a function of applied field. A polynomial background was subtracted and the ALCs were subsequently modelled using the software Quantum, with the eSR modelled using the density matrix formalism described in the previous section [16]. The ALC curve simulations presented in the following are performed considering a muonelectron system. No nuclei are taken into account as their influence was discovered to be negligible [7]. Further details of the modelling can be found elsewhere $[5-7,16]$.

\section{Results and discussion}

Fig. 2 shows the ALCs measured for the three samples at $10 \mathrm{~K}$ and $300 \mathrm{~K}$. In all of the samples, four individual ALCs were observed, although we anticipate there to be six positions on the molecule that the muonium could bond to. There could be several reasons for there being two unobserved ALC resonances. It could be that the formation probability for these two is significantly smaller than the observed resonances, which may simply be related to it not being energetically favourable to bond there. This would result in a small amplitude, resulting in a potentially unobservable ALC resonance. The missing resonances could also have hyperfine coupling parameters that are similar to one or more of the others, resulting in more than one ALC merging or overlapping. Finally, there could be additional ALCs at higher fields that are as-yet undiscovered.

As is expected in the limit of a temperature dependent eSR, the low temperature data have roughly all the same amplitudes $[5,10]$. However, in contrast to the previously published results in the TES and metal Xq3 series [5], the ALC amplitude at $300 \mathrm{~K}$ does not follow a strong trend as the mass of the substituent is increased. Since the amplitude of the ALC is strongly related to the eSR, with the amplitude being roughly proportional to eSR below around $1 \mathrm{MHz}$, this first observation of the spectra suggests that the eSR either slightly falls as a function of increasing mass of the substituent or it remains roughly the same. This is not indicative of a spin orbit mediated mechanism for eSR, as previously reported in similar molecules [5]. Given the complexity of the spectra in Fig. 2, it is more informative to model the resonances to extract the eSR. The extracted hyperfine coupling constants and eSR values for $10 \mathrm{~K}$ and $300 \mathrm{~K}$ are shown in Table 1.

With the same methodology used previously [5-7], we have plotted the average eSR extracted from the ALCs in the ADT series as a function of atomic number, $\mathrm{Z}$, of the

substituted atom (C, Si and Ge). This is shown in the inset to Fig. $3 a$, where it can be seen that there is very little change as a function of $Z$ of the substituted atom, within the error of the measurement. This is inconsistent with the $Z$ dependence of eSR in the $\mathrm{Xq} 3$

and TES series, where a significant rise is observed as the atomic number of the substituent atom is increased, as a result of the spin orbit interaction driving eSR. This inconsistency is relatively easy to explain. It is related to the position of the substituted atom on the ADT molecule (the side group) in relation to the location of the electronic wavefunction, which will be predominantly located on the back bone of the molecule, with very little spin density on the side groups. To demonstrate this further, we have performed DFT calculations of the highest occupied and lowest unoccupied molecular orbitals (HOMO and LUMO, respectively) of the series of molecules, using B3LYP/6-31G(d) with the GAMESS software package [17], shown in Fig. 3(b)-(d). We can come to two conclusions from these calculations. Firstly, there is very little difference in the HOMO or LUMO in the three molecules. Secondly, hardly any spin density exists from the HOMO or LUMO on the side groups, and we would expect that the muonium electron (in a singularly occupied molecular orbital) to be similar. This supports our conclusion that we would not expect there to be much change in the eSR as in the different molecules, even if the underlying mechanism is from the spin orbit interaction. In the previously reported study, the substitution was on the backbone of the TES series and in the centre of the $\mathrm{Xq} 3$ series, so the electronic wavefunction had significant overlap with the substituted atom. It

Table 1

Simulation parameters for the three samples, TBu-ADT, TMS-ADT and TGe-ADT. F is the weighting in \%, and the hyperfine coupling parameters (A: isotropic; D1/D2: anisotropic) and electron spin relaxation rate (eSR) are given in MHz. Errors are estimated from the sensitivity of the simulation on parameters, statistical error bars of the data, and accuracy of the background subtraction.

\begin{tabular}{|c|c|c|c|c|c|c|c|c|c|}
\hline & & \multicolumn{4}{|l|}{$10 \mathrm{~K}$} & \multicolumn{4}{|l|}{$300 \mathrm{~K}$} \\
\hline & & ALC 1 & ALC 2 & ALC 3 & ALC 4 & ALC 1 & ALC 2 & ALC 3 & ALC 4 \\
\hline \multirow[t]{5}{*}{ TBu-ADT } & $F$ & $24 \pm 0.3$ & $28 \pm 0.3$ & $42 \pm 0.3$ & $6 \pm 0.3$ & $24 \pm 0.3$ & $28 \pm 0.3$ & $42 \pm 0.3$ & $6 \pm 0.3$ \\
\hline & $A$ & $107 \pm 1$ & $137 \pm 1$ & $161 \pm 1$ & $201 \pm 1$ & $107 \pm 1$ & $128 \pm 1$ & $154 \pm 1$ & $201 \pm 1$ \\
\hline & $D_{1}$ & $4 \pm 0.1$ & $8 \pm 0.1$ & $10 \pm 0.1$ & $5 \pm 0.1$ & $4 \pm 0.1$ & $6 \pm 0.1$ & $9 \pm 0.1$ & $7 \pm 0.1$ \\
\hline & $D_{2}$ & $5 \pm 0.1$ & $9 \pm 0.1$ & $12 \pm 0.1$ & $5 \pm 0.1$ & $5 \pm 0.1$ & $3 \pm 0.1$ & $13 \pm 0.1$ & $7 \pm 0.1$ \\
\hline & eSR & $0.02 \pm 0$ & $0.02 \pm 0$ & $0.02 \pm 0$ & $0.02 \pm 0$ & $0.02 \pm 0.02$ & $0.15 \pm 0.02$ & $0.82 \pm 0.02$ & $0.78 \pm 0.05$ \\
\hline \multirow[t]{5}{*}{ TMS-ADT } & $F$ & $18 \pm 0.3$ & $25 \pm 0.3$ & $50 \pm 0.3$ & $7 \pm 0.3$ & $18 \pm 0.3$ & $25 \pm 0.3$ & $50 \pm 0.3$ & $7 \pm 0.3$ \\
\hline & $A$ & $107 \pm 1$ & $139 \pm 1$ & $158 \pm 1$ & $201 \pm 1$ & $106 \pm 1$ & $132 \pm 1$ & $153 \pm 1$ & $205 \pm 1$ \\
\hline & $D_{1}$ & $4 \pm 0.1$ & $6 \pm 0.1$ & $10 \pm 0.1$ & $5 \pm 0.1$ & $106 \pm 1$ & $132 \pm 1$ & $153 \pm 1$ & $205 \pm 1$ \\
\hline & $D_{2}$ & $4 \pm 0.1$ & $6 \pm 0.1$ & $9 \pm 0.1$ & $5 \pm 0.1$ & $106 \pm 1$ & $132 \pm 1$ & $153 \pm 1$ & $205 \pm 1$ \\
\hline & eSR & $0.02 \pm 0$ & $0.02 \pm 0$ & $0.02 \pm 0$ & $0.02 \pm 0$ & $0.45 \pm 0.03$ & $0.15 \pm 0.02$ & $0.50 \pm 0.02$ & $0.90 \pm 0.02$ \\
\hline \multirow[t]{5}{*}{ TGe-ADT } & $F$ & $31 \pm 0.3$ & $44 \pm 0.3$ & $18 \pm 0.3$ & $7 \pm 0.3$ & $31 \pm 0.3$ & $44 \pm 0.3$ & $18 \pm 0.3$ & $7 \pm 0.3$ \\
\hline & $A$ & $133 \pm 1$ & $162 \pm 1$ & $180 \pm 1$ & $238 \pm 0.1$ & $130 \pm 1$ & $151 \pm 1$ & $178 \pm 1$ & $288 \pm 1$ \\
\hline & $D_{1}$ & $7 \pm 0.1$ & $11 \pm 0.1$ & $9 \pm 0.1$ & $10 \pm 0.1$ & $6 \pm 0.1$ & $9 \pm 0.1$ & $7 \pm 0.1$ & $8 \pm 0.1$ \\
\hline & $D_{2}$ & $7 \pm 0.1$ & $9 \pm 0.1$ & $4 \pm 0.1$ & $11 \pm 0.1$ & $3 \pm 0.1$ & $9 \pm 0.1$ & $8 \pm 0.1$ & $11 \pm 0.1$ \\
\hline & eSR & $0.02 \pm 0$ & $0.02 \pm 0$ & $0.02 \pm 0$ & $0.02 \pm 0$ & $0.20 \pm 0.02$ & $0.32 \pm 0.02$ & $0.35 \pm 0.02$ & $0.50 \pm 0.05$ \\
\hline
\end{tabular}



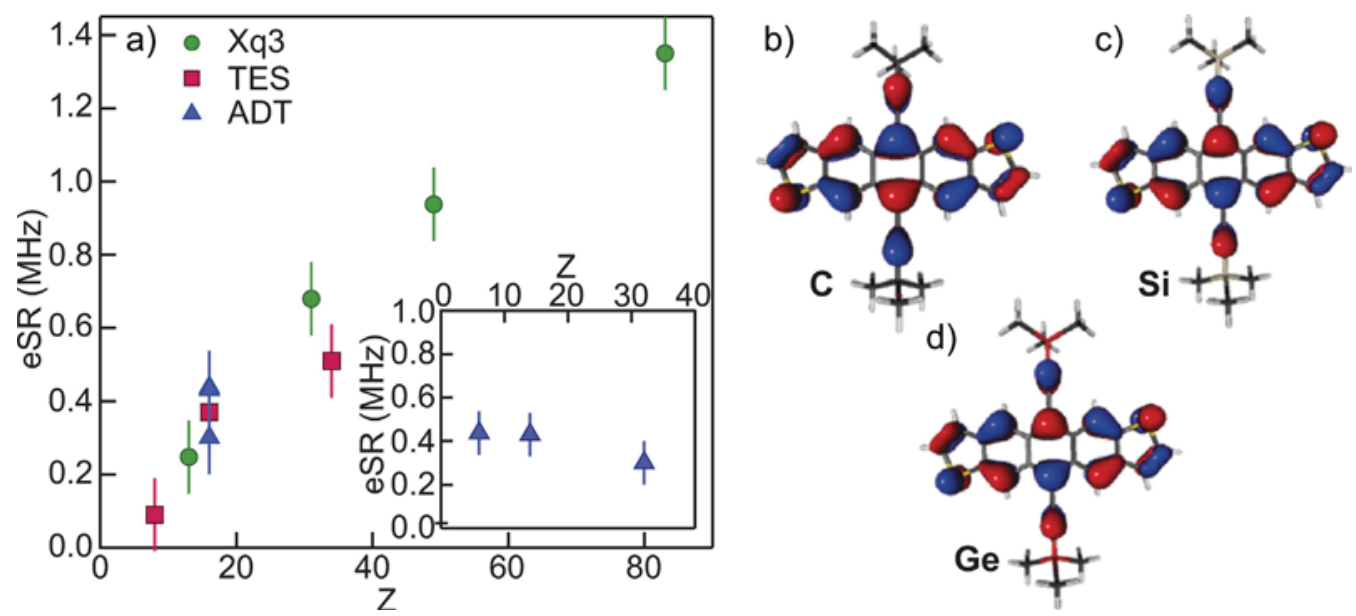

Fig. 3. (a) The average eSR of the ADT series plotted against the atomic number of the back-bone heaviest atom (Sulphur), which shows excellent agreement with the previously published trend in the average eSR of two other organic semiconductors [5]. Inset: no Z dependence of the average eSR is observed when plotted against the substituted atom of the ADT series. (b)-(d) The HOMO and LUMO levels of the three molecules in the series.

is therefore more appropriate to plot this against the atomic number of the heaviest atom on the backbone, in this case $\mathrm{S}$, as shown in the main panel of Fig. 3(a). This fits very nicely on the trend.

\section{Conclusions}

We have reported eSR measurements on a molecular series based on an anthradithiophene, with targeted chemical substitution on the side groups. We found very little change in the average eSR as the mass of the substituent atom increases, which can be explained by the electronic wavefunction being mainly located on the backbone of the molecule, which does not undergo any changes in the series. There is therefore very little electronic wavefunction overlap with the substituted atom on the side group, such that substituting one of the atoms with a heavier species does not significantly increase the spin orbit interaction, which has been shown to be a very effective mechanism of eSR in organic molecules [5].

\section{Acknowledgements}

AJD would like to acknowledge financial support from the UK Engineering and Physical Sciences Research Council, grant number EP/G054568/1, the European Union Seventh Framework Programme project NMP3-SL-2011-263104 'HINTS' and the European Research Council project 'Muon Spin Spectroscopy of Excited States (MuSES)' proposal number 307593. SZ and LS acknowledge financial support from the National Science Foundation of China, grant numbers 61307039 and 61404088 .

\section{References}

[1] S. Sanvito, Injecting and controlling spins in organic materials, J. Mater. Chem. 17 (2007) 4455-4459.

[2] A.J. Drew, J. Hoppler, L. Schulz, F.L. Pratt, P. Desai, P. Shakya, T. Kreouzis, W.P. Gillin, A. Suter, N.A. Morley, V.K. Malik, A. Dubroka, K.W. Kim, H. Bouyanfif, F. Bourqui, C. Bernhard, R. Scheuermann, G.J. Nieuwenhuys, T. Prokscha, E. Morenzoni, Direct measurement of the electronic spin diffusion length in a fully functional organic spin valve by low-energy muon spin rotation, Nat. Mater. 8 (2009) 109-114.

[3] L. Schulz, L. Nuccio, M. Willis, P. Desai, P. Shakya, T. Kreouzis, V.K. Malik, C. Bernhard, F.L. Pratt, N.A. Morley, A. Suter, G.J. Nieuwenhuys, T. Prokscha, E. Morenzoni, W.P. Gillin, A.J. Drew, Engineering spin propagation across a hybrid organic/inorganic interface using a polar layer, Nat. Mater. 10 (2011) 39-44.

[4] A.J. Drew, F.L. Pratt, J. Hoppler, L. Schulz, V. Malik-Kumar, N.A. Morley, P. Desai, P. Shakya, T. Kreouzis, W.P. Gillin, K.W. Kim, A. Dubroka, R. Scheuermann, Intrinsic mobility limit for anisotropic electron transport in $\mathrm{Alq}_{3}$, Phys. Rev. Lett. 100 (2008) 116601.

[5] L. Nuccio, M. Willis, L. Schulz, S. Fratini, F. Messina, M. D'Amico, F.L. Pratt, J.S. Lord, I. McKenzie, M. Loth, B. Purushothaman, J. Anthony, M. Heeney, R.M. Wilson, I. Hernández, M. Cannas, K. Sedlak, T. Kreouzis, W.P. Gillin, C. Bernhard, A.J. Drew, Importance of spin-orbit interaction for the electron spin relaxation in organic semiconductors, Phys. Rev. Lett. 110 (2013) 216602.

[6] L. Nuccio, M. Schulz, F.L. Willis, M. Pratt, N. Heeney, C. Stingelin, A.J. Drew, Electron spin relaxation in organic semiconductors probed, SR, J. Phys.: Conf. Ser. 292 (12) (2011) 004

[7] L. Schulz, M. Willis, L. Nuccio, P. Shusharov, S. Fratini, F.L. Pratt, W.P. Gillin, T. Kreouzis, M. Heeney, N. Stingelin, C.A. Stafford, D.J. Beesley, C. Bernhard, J.E. Anthony, I. McKenzie, J.S. Lord, A.J. Drew, Importance of intramolecular electron spin relaxation in small molecule semiconductors, Phys. Rev. B 84 (2011) 085209.

[8] S.J. Blundell, Muon-spin rotation studies of electronic properties of molecular conductors and superconductors, Chem. Rev. 104 (2004) 5717-5735.

[9] Muon Science, in: S.L. Lee, S.H. Kilcoyne, R. Cywinski (Eds.), first ed., The Scottish Universities Summer School in Physics, 1999.

[10] L. Nuccio, L. Schulz, A.J. Drew, Muon spin spectroscopy: magnetism, soft matter and the bridge between the two, J. Phys. D: Appl. Phys. 47 (2014) 473001.

[11] B.D. Patterson, Muonium states in semiconductors, Rev. Mod. Phys. 60 (1988) 69-159.

[12] P.F. Meier, Spin dynamics of transitions between muon states, Phys. Rev. A 25 (1982) 1287.

[13] M.M. Payne, S.A. Odom, S.R. Parkin, J.E. Anthony, Stable, crystalline acenedithiophenes with up to seven linearly fused rings, Org. Lett. 6 (2004) $3325-3328$.

[14] J.E. Anthony, B. Purushothaman, Crystal design for organic semiconductors: the effects of substitution on crystal packing, organic field- effect transistors, (SPIE, Bellingham, WA), SPIE Proceedings, 66582007, pp. 6580L.

[15] F.L. Pratt, WIMDA: a muon data analysis program for the Windows PC, Physica B 289 (2000) 710-7714.

[16] J.S. Lord, Computer simulation of muon spin evolution, Physica B 374 (2006) $472-474$.

[17] M.W. Schmidt, K.K. Baldridge, J.A. Boatz, S.T. Elbert, M.S. Gordon, J.H. Jensen, S. Koseki, N. Matsunaga, K.A. Nguyen, S. Su, T.L. Windus, M. Dupuis, J.A. Montgomery Jr., General atomic and molecular electronic structure system, J. Comput. Chem. 14 (1993) 1347-1363. 